\title{
ISMAAR: Leading the global agenda for a more physiological, patient-centred, accessible and safer approaches in ART
}

\author{
Geeta Nargund • R. C. Chian
}

Published online: 21 February 2013

(C) Springer Science+Business Media New York 2013

Geeta Nargund UK; President, ISMAAR

RC Chian, Canada: Board member ISMAAR and On behalf

of Organising Committee ISMAAR Congress 2013

The International Society for Mild Approaches in Assisted Reproduction (ISMAAR) is a UK registered Charity (non-profit making organisation) founded to promote safer, less drug-orientated, more physiological, widely accessible and woman-friendly approaches in assisted reproduction. Since its inception, the society has conducted five successful world congresses and several satellite workshops in developing countries. ISMAAR terminology for stimulation protocols in IVF [1] was published in Human Reproduction in 2007. ISMAAR prides itself in having internationally renowned scientists and clinicians on its Board who are dedicated to the objectives of ISMAAR. We believe this approach is much needed based on growing concerns about the lack of wider accessibility of assisted reproductive treatments globally and the need to put the welfare of women at the top of the agenda in Assisted Reproduction Technology (ART) [2]. Thus, ISMAAR aims to accomplish the following:

\section{Reducing cost and increasing accessibility of ART}

The 6th World Congress held in Nanjing, China, is an opportune time to discuss the role of natural, mild IVF and IVM including simplified laboratory systems in reducing costs and increasing accessibility in developing countries. Mild IVF will allow more patients to be treated, at lower cost and with higher patient safety and acceptability in both the developing and the developed world [3].

\section{Patient-centred approaches}

The current conventional IVF protocols can be complex, aggressive, unphysiological, unfriendly, unnecessary, and unregulated in many countries. The treatment involves pituitary downregulation followed by higher daily doses of stimulation lasting for 4 to 5 weeks. As a result, women may experience considerable discomfort [3]. Milder approaches fit within women's natural cycles and are associated with less burden and drop outs from treatment. [4].

\section{OHSS-Free IVF}

Although the reported incidence of severe OHSS is around $2 \%$, several reports suggest a higher rate of hospitalisation. Recent reports concerning maternal deaths related to OHSS in the UK and Netherlands suggest 3 deaths per 100,000 IVF cycles performed [5]. There is no global registry reporting deaths due to OHSS. Modern protocols using Gn RH analogue for trigger in antagonist cycles is the way forward to run an OHSS-free service. [5]. Mild stimulation protocols conducted in antagonist cycles facilitate the introduction of "OHSS-free clinics" which needs to be a top priority in IVF practice. 


\section{Healthier oocytes, embryos and potentially higher birth weight babies}

The primary aim of mild stimulation is a more physiological approach to stimulation with the collection of fewer mature oocytes. Studies have shown than this approach may be beneficial for oocyte/embryo quality [6] and endometrial receptivity [7]. Furthermore, there is preliminary evidence than the mean birth weight of the child is higher when in modified natural cycles compared to conventional stimulated cycles. [8].

\section{Fertility preservation}

Immature oocyte collection from ovaries can be perfomed both in follicular and luteal phase without ovarian stimulation. In vitro maturation (IVM) of oocytes followed by vitrification offers a realistic and safer method for fertility preservation in cancer patients [9]. The role of IVM in women desirous of having Natural IVF treatment is an important area for future research.

\section{Summary}

Mild approaches in assisted reproduction are at the forefront of new developments in IVF practice. Further prospective randomised studies are required. The obligation to make ART safer, affordable and patient-friendly is upon us and
ISMAAR is committed to making this happen. The papers published in this special issue would represent a significant step forward.

\section{References}

1. Nargund G, Fauser BC, Macklon NS, Ombelet W, Nygren K, Frydman R. The ISMAAR proposal on terminology for ovarian stimulation for IVF. Hum Reprod. 2007;22:2801-4.

2. Nargund G, Frydman R. Towards a more physiological approach to IVF. Reprod BioMed Online. 2007;14(5):550-2.

3. Macklon NS, Stouffer RL, Giudice LC. The science behind 25 years of ovarian stimulation for in vitro fertilization. Endocr Rev. 2006;27:170-207.

4. Verberg MF, Eijkemans MJ, Heijnen EM, Broekmans FJ, De Klerk C, Fauser BC. Why do couples drop-out from IVF treatment? A prospective cohort study. Hum Reprod. 2008;23:2050-5.

5. Devroey P, Polyzos N, Blockeel C. An OHSS-Free Clinic by segmentation of IVF treatment. Hum Reprod. 2011;26:2593-7.

6. Baart EB, Martini E, Eijkemans J, et al. Milder ovarian stimulation for in-vitro fertilisation reduces aneuolpidy in the human preimplantation embryo: a randomised controlled trial. Human Reprod 1-9 2007.

7. Devroey P, Bourgain C, Macklon NS, Fauser BCJM. Reproductive biology and IVF: ovarian stimulation and endometrial receptivity. Trends Endocrinol Metab. 2004;15:84-90.

8. Pelinck MJ, Keizer MH, Hoek A, et al. Perinatal outcome in singletons after modified natural cycle IVF and standard IVF with ovarian stimulation; Eur J Obste Gynaecol. Reprod Biol. 2010;148(1):5661.

9. Chian RC, Uzelac P, Nargund G. In Vitro Maturation of human immature oocytes for fertility preservation. Fertil Steril (in press) 